\title{
Integrated Silicon-Organic Hybrid (SOH) Frequency Shifter
}

\author{
M. Lauermann ${ }^{1}$, C. Weimann ${ }^{1}$, A. Knopf ${ }^{2}$, D. L. Elder ${ }^{3}$, W. Heni' ${ }^{1}$, R. Palmer ${ }^{1}$, D. Korn ${ }^{1}$, P. C. Schindler ${ }^{1}$, \\ S. Koeber ${ }^{1}$, L. Alloatti ${ }^{1}$, H. Yu ${ }^{4,5}$, W. Bogaerts ${ }^{4}$, L. R. Dalton ${ }^{3}$, C. Rembe ${ }^{2}$, J. Leuthold ${ }^{1,6}$, W. Freude ${ }^{1}$, C. Koos ${ }^{1}$ \\ ${ }^{1}$ Karlsruhe Institute of Technology (KIT), Institutes IPQ and IMT, Engesserstr. 5, 76131 Karlsruhe, Germany \\ ${ }^{2}$ Polytec GmbH, 76337 Waldbronn, Germany \\ ${ }^{3}$ University of Washington, Department of Chemistry, Seattle, WA 98195-1700, United States \\ ${ }^{4}$ Ghent University - IMEC, Photonics Research Group, Gent, Belgium \\ ${ }^{5}$ now with: Department of Information Science and Electronic Engineering, Zhejiang University, Hangzhou 310027, China \\ ${ }^{6}$ Electromagnetic Fields Laboratory, Swiss Federal Institute of Technology (ETH), Zurich, Switzerland \\ matthias.lauermann@kit.edu,christian.koos@kit.edu
}

\begin{abstract}
We demonstrate a waveguide-based frequency shifter on the silicon-organic hybrid (SOH) platform, enabling frequency shifts up to $10 \mathrm{GHz}$. Spurious side-modes are suppressed by more than $23 \mathrm{~dB}$ using temporal shaping of the drive signal.

OCIS codes: (250.4110) Modulators; (060.2630) Frequency modulation
\end{abstract}

\section{Introduction}

Integrated electro-optic frequency shifters are key elements for various applications such as interferometric metrology and are also highly interesting for optical communications [1,2]. Currently, acousto-optic modulators $(\mathrm{AOM})$ are used for frequency shifting, providing high side-mode suppression ratio (SMSR) and conversion efficiency (CE). However, operation frequencies of these devices are limited, and integration into planar waveguide circuits proves to be difficult, especially when material systems with vanishing second-order nonlinearities such as silicon-on-insulator (SOI) are to be used as an integration platform. On the other hand, waveguide-based phase modulators are standard building blocks in silicon photonics. Serrodyne frequency shifters can be realized by driving a single phase modulator with a sawtooth signal, but this concept poses stringent requirements on the electrical drive signal to obtain large SMSR and good CE [3]. A serrodyne frequency shifter has recently been demonstrated on the silicon photonic platform [4], resulting in a remarkable SMSR of $39 \mathrm{~dB}$, while the frequency shift was limited to $1 \mathrm{kHz}$ due to the speed of the deployed thermo-optic phase shifters. Another approach is based on combining the output of two Mach Zehnder modulators (MZM) with a $\pi / 2$ phase shift, hence realizing a device that acts independently on the in-phase and quadrature component of the optical signal. When driving the two MZM of such an IQ modulator by a sine and a cosine signal, frequency shifting by means of single side band (SSB) modulation can be achieved [5, 6]. However, this concept suffers from a trade-off between SMSR and CE: For high SMSR, the MZM need to be driven in the small-signal regime, where the amplitude transmission depends linearly on the applied voltage. High $\mathrm{CE}$, on the other hand, requires large signals to drive the device to maximum transmission, but comes along with spurious side-modes in the optical spectrum due to the nonlinear transfer function of the MZM. Moreover, SSB modulation with high SMSR is hard to achieve with state-of-the-art silicon photonic devices: Current phase modulators are based on free-carrier dispersion in SOI waveguides, which leads to an inherent coupling of amplitude and phase modulation and thereby also generates unwanted side-modes.

In this paper, we demonstrate that SSB frequency shifters can be realized on the silicon photonic platform. We use silicon-organic hybrid ( $\mathrm{SOH}$ ) phase modulators that combine SOI slot waveguides with electro-optic (EO) organic materials, thereby enabling efficient and broadband phase modulation without introducing unwanted amplitude-phase coupling [7, 8]. Using SOH phase modulators of only $1 \mathrm{~mm}$ length, we demonstrate frequency shifting of up to $10 \mathrm{GHz}$. Furthermore we introduce a concept to overcome the trade-off between SMSR and CE by temporal shaping of the drive signal. We experimentally show more than $23 \mathrm{~dB}$ SMSR and a CE of $-5.8 \mathrm{~dB}$.

\section{Principle of SOH frequency shifter}

A schematic of a SOH MZM is depicted in Fig. 1(a). In both arms of the MZM, light is guided in the silicon slot waveguides $\left(w_{\text {Slot }}=140 \mathrm{~nm}, w_{\text {Rail }}=220 \mathrm{~nm}, h_{\text {Rail }}=220 \mathrm{~nm}\right)$ which are filled with an organic electro-optic material. Due to the index contrast between the silicon rails $(n=3.48)$ and the organic cladding $(n=1.83)$, the electrical field of the fundamental quasi-TE mode is enhanced in the slot. The modulating electric field is applied to the slot waveguides via a ground-signal-ground (GSG) coplanar waveguide connected to a conductive silicon slab with a thickness of $50 \mathrm{~nm}$. An applied electrical voltage drops mainly across the slot, leading to a high overlap of the modulating field with the optical mode. A detailed description of the device can be found in [9]. To align the EO chromophores in the slot, the material is poled by applying an electric field at elevated temperatures across the ground electrodes, thus orienting the molecules in both MZM arms in the same direction, indicated by green arrows in Fig. 1(a). Applying a modulating signal to the GSG electrodes leads to an electric field pointing outwards from the center electrode (red arrows) and hence to opposite phase shifts in both arms, which corresponds to push-pull 

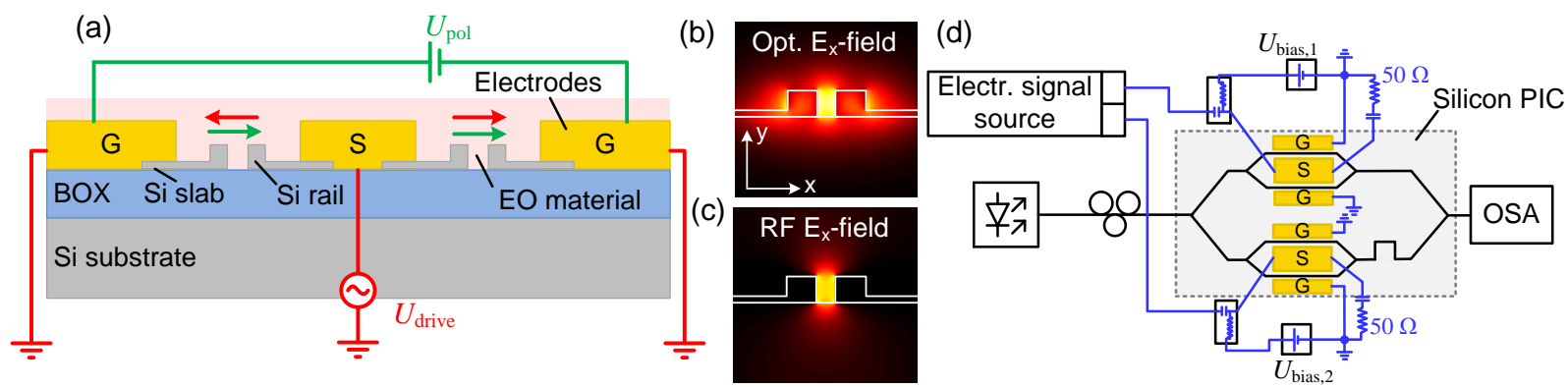

Fig. 1(a) Cross-sectional schematic of an SOH MZM. The slot waveguides are filled with the electro-optic organic material DLD164. Electrical contact is established via a conductive silicon slab to an RF transmission line, which is impedance-matched to the signal generator. The chromophores in the slot have to be oriented by a poling voltage $U_{\text {pol }}$, applied to the outer ground electrodes of the MZM. Due to the electric field in the slot the chromophores in both waveguides are oriented in the same global direction. The driving voltage $U_{\text {drive }}$ is applied via the GSG line and leads to opposite phase shifts in both arms (push-pull operation). (b) Dominant $E_{\mathrm{x}}$ component of the optical field and (c) $E_{\mathrm{x}}$ component of the electrical drive signal in the slot waveguide. Both fields are confined to the slot, resulting in strong interaction and hence efficient modulation. (d) Schematic of the experimental setup. A tunable laser (emission wavelength around $1550 \mathrm{~nm}$ ) is used as optical source and coupled to the silicon chip via a grating coupler. The on-chip IQ modulator comprises two nested SOH MZM. An intentional imbalance of $40 \mu \mathrm{m}$ is placed between the two MZM to adjust the $\pi / 2$ phase shift via the wavelength. The output signal is recorded by an OSA with $20 \mathrm{MHz}$ resolution bandwidth.

operation. The cladding consists of the strongly electro-optic chromophore DLD164 [10], with an in-device EO coefficient of $180 \mathrm{pm} / \mathrm{V}$. This leads to a remarkably low voltage-length product of $U_{\pi} L=0.6 \mathrm{Vmm}$ in the DC limit. For SSB operation, two Mach-Zehnder modulators operated in push-pull mode are nested to an IQ modulator as shown in Fig. 1(d).

\section{Experimental demonstration}

A sketch of the measurement setup is depicted in Fig. 1(d). The bias voltage for each MZM is coupled to the chip via a bias-T and adjusted for zero transmission in the absence of any modulation signal. The optical spectrum at the output is recorded by an optical spectrum analyzer (OSA) with $20 \mathrm{MHz}$ resolution bandwidth. In a first experiment, we use an RF synthesizer with a large frequency range as electrical source to operate the device in the small-signal regime. For SSB modulation, two sinusoidal drive signals with the same frequency $\Omega$ and a $\pi / 2$ phase difference are applied to the MZM. With an optical input at frequency $\omega$, the field at the output should only consist of odd harmonics at the frequencies $\omega+n \Omega$, where $n=(-1,3,-5,7 \ldots)$. The amplitude of the sideband at frequency $n \Omega$ is described by the Bessel function $J_{n}(m)$, where $m=\pi \cdot U_{\mathrm{p}} /\left(2 U_{\pi}\right)$ denotes the modulation index and $U_{\pi}$ is the $\pi$-voltage of the push-pull MZM [6].

The optical output spectrum at $\Omega=0.475 \mathrm{GHz}$ is depicted in Fig. 2(a). By fine-tuning the bias voltage, the carrier can be suppressed by $37 \mathrm{~dB}$. The shifted line at $\omega-\Omega$ is clearly visible with a CE of $-16.4 \mathrm{~dB}$ and SMSR of $18.75 \mathrm{~dB}$. It has to be noted that the strongest spurious lines occur at $\omega \pm 2 \Omega$ rather than at $\omega+3 \Omega$, as would be expected [6]. This is attributed to slightly different poling efficiencies of the two phase shifters in the arms of the two MZM. The ratio of the efficiency of the phase shifters in a single MZM can be estimated from these side lines to be 1/0.8. This can be overcome by improving the poling procedure or by using dual drive configurations of the MZM. By increasing the modulation frequency and simultaneously adjusting the drive voltage, we can compensate the decaying transfer function of the device and demonstrate frequency shifts of up to $10 \mathrm{GHz}$ without significant degradation of SMSR, Fig. 2(b). We expect that the operation bandwidth and the flatness of the frequency response
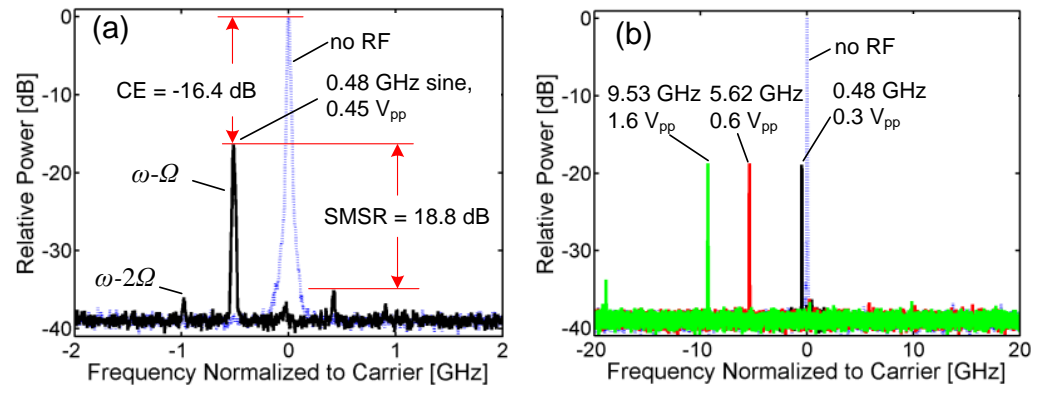

Fig. 2(a) The spectrum of the optical output with a sinusoidal RF signal applied $\left(\Omega=0.475 \mathrm{GHz}\right.$ and $U_{\text {drive }}=0.45 \mathrm{~V}_{\mathrm{pp}}$ per MZM). The bias point of both MZM and the phase relation of the optical as well as the electrical paths is chosen such to minimize the carrier and unwanted frequency components. In blue the spectra of the optical carrier is plotted, without RF signal and the bias set to maximum transmission. The conversion efficiency (CE) is $-16.38 \mathrm{~dB}$ and the side-mode suppression ratio (SMSR) is $18.75 \mathrm{~dB}$. (b) Output spectra for different RF frequencies. Operation in a wide bandwidth is possible, for larger frequencies of the drive signal, higher RF voltage is needed to keep the power in the shifted line constant. 

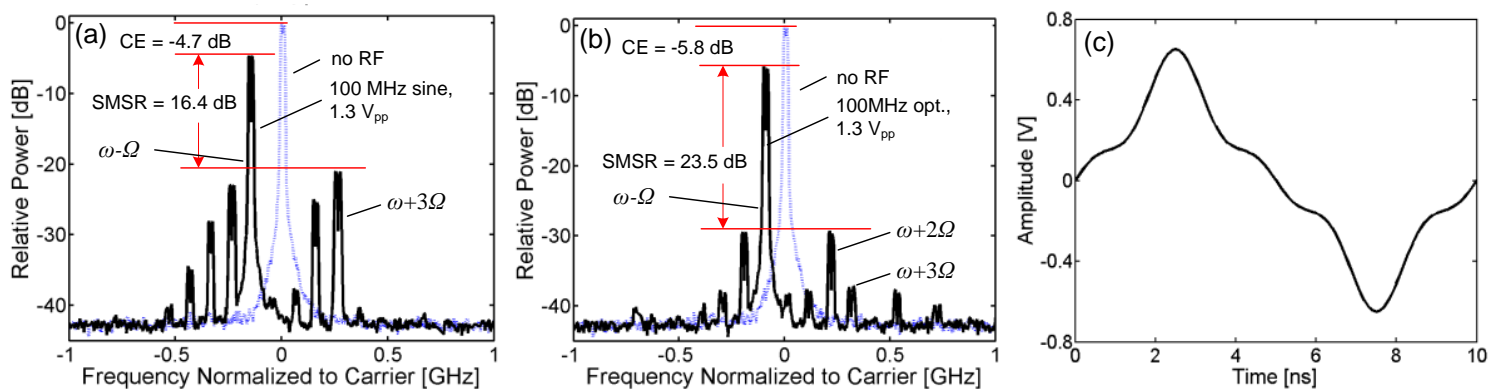

Fig. 3: The frequency shifter is driven with an AWG at $\Omega=100 \mathrm{MHz}$, the voltage of $1.3 \mathrm{~V}_{\mathrm{pp}}$ at the MZM is optimized for maximum conversion. Each MZM of the frequency shifter is controlled separately by one channel of the AWG in terms of amplitude, phase relation and shape of the waveform. (a) A plot of the output spectrum with a pure sinusoidal drive signal, resulting in an optical spectrum with good CE but strong harmonics. (b) shows the output with additional harmonics in the drive signal which suppress the unwanted harmonics in the output spectrum. With the optimization the spurious component at $\omega+3 \Omega$ could be reduced by over $15 \mathrm{~dB}$, the overall SMSR is $23.5 \mathrm{~dB}$. The waveform of the optimized drive signal for one MZM can be seen in (c), it was generated using $U=\sin (\Omega t)-0.25 \sin (3 \Omega t)+0.125 \sin (5 \Omega t)$.

can be significantly improved by optimized device designs, enabling a $3 \mathrm{~dB}$ bandwidth of tens of $\mathrm{GHz}$ [8].

To increase CE, a higher modulation index $m$ is necessary. To suppress spurious modes associated with large-signal operation of the MZM, we use periodic drive signals which comprise higher uneven harmonics with carefully optimized amplitudes. These harmonics lead to a deformation of the drive signal, which, in combination with the nonlinear response of the MZM, generates a sinusoidal amplitude modulation at the output of each MZM. In theory, all remaining uneven lines in the optical output signal can hence be suppressed by choosing the right combination of harmonics. For ideal components, this would lead to a triangular drive signal, peaking at $\pm U_{\pi}$ of the push-pull MZM. To verify this concept, we use an arbitrary waveform generator (AWG) with two channels to drive the device. Keeping the frequency at $\Omega=100 \mathrm{MHz}$, the drive amplitude, waveform and electrical phase relation of each singledrive MZM can be controlled. In Fig. 3(a) a purely sinusoidal signal is fed into the frequency shifter with a large amplitude of approximately $U_{\pi}$, optimized for maximum power in the $\omega-\Omega$ line. This results in a CE of $-4.7 \mathrm{~dB}$ and an SMSR of only $16.4 \mathrm{~dB}$, in good agreement with the theory and previous results in LiNbO3 (CE of $-4.7 \mathrm{~dB}$ and an SMSR of $18.4 \mathrm{~dB},[5])$. We then use an experimentally optimized drive signal comprising the third and fifth harmonic, $U=\sin (\Omega t)-0.25 \sin (3 \Omega t)+0.125 \sin (5 \Omega t)$, see Fig. 3(c) for the time-domain electrical waveform. This significantly reduces the spurious side-modes in the optical spectrum, Fig. 3(b). The previously dominant line at $\omega+3 \Omega$, targeted by the third harmonic in the drive signal, is $31 \mathrm{~dB}$ below the line at $\omega-\Omega$. The $\mathrm{CE}$ amounts to $-5.8 \mathrm{~dB}$, quite close to the theoretically predicted value of $-6 \mathrm{~dB}$, while the SMSR has improved to $23.5 \mathrm{~dB}$ and is now limited by the spurious lines at $\omega \pm 2 \Omega$. These lines originate from the unequal efficiency of the phase shifters in the MZM and can be avoided by improved device design. In principle, a frequency shifter with over $30 \mathrm{~dB}$ SMSR and approximately $6 \mathrm{~dB}$ conversion loss is possible, using the SSB configuration combined with temporal shaping of the drive signal.

\section{Summary}

We have demonstrated an SSB frequency shifter in a silicon photonic circuit, realized by SOH integration. The device allows for broadband operation up to $10 \mathrm{GHz}$. Temporal shaping of the electrical drive signal enables good CE of $-5.8 \mathrm{~dB}$ while maintaining high SMSR of more than $23 \mathrm{~dB}$. The SMSR can be improved further by using optimized device designs or dual-drive configuration of the modulators.

We acknowledge support by the European Research Council (ERC Starting Grant 'EnTeraPIC', number 280145), the EU-FP7 projects SOFI, OTONES, and PHOXTROT, the Alfried Krupp von Bohlen und Halbach Foundation, the Karlsruhe International Research School for Teratronics (HIRST), the Karlsruhe School of Optics and Photonics (KSOP), the DFG Center for Functional Nanostructures (CFN), and the Karlsruhe Nano-Micro Facility (KNMF). We acknowledge financial support of The National Science Foundation (DMR-0905686, DMR0120967) and the Air Force Office of Scientific Research (FA9550-09-1-0682).

\section{References}

[1] Y. Ma, et al., Optics express, vol. 17, pp. 9421-9427, 2009.

[2] H. Yamazaki, et al., Optics express, vol. 19, pp. B69-B74, 2011.

[3] L. M. Johnson, et al., J. Lightw. Technol., vol. 6, pp. 109-112, 1988.

[4] Y. Li, et al., in 7th IEEE Group IV Photonics, 2010, pp. 75-77.

[5] S. Shimotsu, et al., IEEE Photon. Technol. Lett., vol. 13, pp. 364366,2001
[6] M. Izutsu, et al., IEEE J. Quantum Electron., vol. 17, pp. 22252227, 1981.

[7] L. Alloatti, et al., Optics express, vol. 19, pp. 11841-11851, 2011.

[8] J. Leuthold, et al., IEEE J. Sel. Topics Quantum Electron., vol. 19, pp. 3401413-3401413, 2013

[9] D. Korn, et al., Optics express, vol. 21, pp. 13219-13227, 2013.

[10]R. Palmer, in 39th European Conf. Opt. Commun., We.3.B.3, London, UK, 2013. 